\title{
Role of sublingual asenapine in treatment of schizophrenia
}

\author{
This article was published in the following Dove Press journal: \\ Neuropsychiatric Disease and Treatment \\ 25 May 20II \\ Number of times this article has been viewed
}

\section{Leslie Citrome}

New York University School of Medicine, Department of Psychiatry, New York, NY, USA
Correspondence: Leslie Citrome I I Medical Park Drive, Suite 106, Pomona, NY 10970

Tel + I $845362208 \mid$

Fax +l 8453628745

Email nntman@gmail.com

\begin{abstract}
Asenapine tablets are a new option for the treatment of schizophrenia. Sublingual administration is essential because bioavailability if ingested is less than $2 \%$. Efficacy is supported by acute and long-term randomized controlled studies conducted by the manufacturer, with asenapine $5 \mathrm{mg}$ twice daily evidencing superiority over placebo in six-week studies of acute schizophrenia, and flexibly-dosed asenapine (modal dose $10 \mathrm{mg}$ twice daily) superior to placebo in a 26-week maintenance of response study. Tolerability advantages over some second-generation antipsychotics, such as olanzapine, include a relatively favorable weight and metabolic profile, as demonstrated in a 52-week randomized, head-to-head, double-blind clinical trial. Although dose-related extrapyramidal symptoms and akathisia can be present, the frequency of these effects is lower than that for haloperidol and risperidone. Somnolence may also occur, and appears to be somewhat dose-dependent when examining rates of this among patients receiving asenapine for schizophrenia and bipolar disorder. Prolactin elevation can occur, but at a rate lower than that observed for haloperidol or risperidone. Unique to asenapine is the possibility of oral hypoesthesia, occurring in about $5 \%$ of participants in the clinical trials. Obstacles to the use of asenapine are the recommendations for twice-daily dosing and the need to avoid food or liquids for 10 minutes after administration, although the bioavailability is only minimally reduced if food or liquids are avoided for only two minutes.
\end{abstract}

Keywords: antipsychotic, asenapine, clinical trials, schizophrenia

\section{Introduction}

Schizophrenia is a relatively common mental disorder that is usually chronic in nature. Interventions are ordinarily targeted to positive psychotic symptoms such as hallucinations or delusions, but negative, cognitive, and mood symptoms can also be disabling. There is an array of antipsychotic medications currently available, distinguishable principally by their safety and tolerability profiles, although some differences in efficacy also exist when groups of patients are compared in randomized controlled trials. ${ }^{1}$ The individual person with schizophrenia may respond to, or tolerate, one antipsychotic but not another, in a manner that is not predictable, lending to empirical trials of different agents when treating the illness in the clinic. The quest is to identify a particular medication that works "well enough", is tolerated "well enough", and that the patient is willing to adhere to.

Asenapine was initially approved by the US Food and Drug Administration (FDA) in 2009 for the treatment of acute schizophrenia and acute manic or mixed episodes associated with bipolar I disorder in adults, and subsequently received approval for the maintenance phase of schizophrenia and for adjunctive use with lithium or valproate 
for acute manic or mixed episodes associated with bipolar I disorder. ${ }^{2}$ A literature search using the key word "asenapine" with no limitations or constraints was undertaken on April 6, 2011 using PubMed (http://www.ncbi.nlm.nih.gov/pubmed/) and Embase (http://www.embase.com/), yielding 67 and 212 records, respectively. Abstracted from the search results were all studies or analyses of asenapine for the treatment of schizophrenia. The PubMed search resulted in five clinical trial reports involving patients with schizophrenia, ${ }^{3-8}$ including a QT study. ${ }^{7,8}$ The Embase search produced six additional relevant citations to abstracts from presentations at medical conferences. ${ }^{9-14}$ Abstracts from the most recent meeting of the American College of Neuropsychopharmacology, held on December 5-9, 2010 were also accessed as published, resulting in three additional citations. ${ }^{15-17}$ Also queried was http://www.clinicaltrials.gov for completed trials with results available on the website for asenapine in patients with schizophrenia. ${ }^{18-24}$ This review is based on those reports and the contents of the most current US product labeling, ${ }^{2}$ together with additional information that can be found in the author's prior review, ${ }^{25}$ as well as in documents available on the FDA website. ${ }^{26-28}$ In total, six short-term and seven longer-term randomized controlled studies of asenapine for the treatment of schizophrenia are included in this review, as outlined in Tables 1 and 2 .

\section{Pharmacokinetics and pharmacodynamics}

Asenapine is unique among the currently available antipsychotics in that it is a fast-dissolving tablet that is absorbed primarily in the oral mucosa. If swallowed, the bioavailability of asenapine is limited to less than $2 \%$ because of high hepatogastrointestinal first-pass metabolism. ${ }^{2,26}$ Patient instructions are to place the tablet under the tongue and not to eat or drink for 10 minutes after administration. ${ }^{2}$ The highly porous tablets, available in $5 \mathrm{mg}$ and $10 \mathrm{mg}$ strengths, dissolve in the saliva within approximately 10 seconds, and result in bioavailability of approximately $35 \% .^{2,26,27}$ Drinking water sooner than 10 minutes after administration of sublingual asenapine can reduce its bioavailability, ${ }^{2,26}$ with a $19 \%$ reduction in plasma exposure observed following water administration at two minutes. If placed elsewhere in the oral cavity, asenapine will still be absorbed, as demonstrated in an open-label, randomized, three-way crossover trial among healthy men who received single $5 \mathrm{mg}$ doses of asenapine via sublingual, supralingual, and buccal routes, ${ }^{29}$ where with buccal administration (ie, "cheeking") the plasma level exposure was almost $25 \%$ higher than for the sublingual route, and $6 \%$ lower with supralingual administration than with sublingual administration. These differences in exposure are smaller than the overall variability observed in studies, where overall exposure varied by $37 \%$, with a mean interindividual variability of $26 \%$ and a mean intraindividual variability of $26 \%{ }^{26}$ Increasing the dose from $5 \mathrm{mg}$ to $10 \mathrm{mg}$ twice daily (a two-fold increase) results in less than linear (1.7 times) increases in both the extent of exposure and maximum concentration. $^{2}$

Peak plasma levels occur rapidly, within 30-90 minutes. ${ }^{2,26,27}$ The mean terminal half-life is approximately 24 hours. $^{2}$ Metabolism is primarily through direct glucuronidation by UGT1A4 and oxidative metabolism by cytochrome P450 (CYP) isoenzymes (predominantly CYP1A2). ${ }^{2}$ Asenapine has a large volume of distribution (approximately $20-25 \mathrm{~L} / \mathrm{kg}$ ) and is highly bound (95\%) to plasma proteins, including albumin and $\alpha 1$-acid glycoprotein. ${ }^{2}$ Despite the fact that smoking can induce CYP1A2, concomitant smoking had no substantial effect on the pharmacokinetics of asenapine when tested in healthy male subjects. ${ }^{2}$ Fluvoxamine, a potent CYP1A2 inhibitor, can increase exposure to asenapine by $29 \%,{ }^{2}$ and should be coadministered with caution. ${ }^{2}$ Asenapine is not recommended in patients with severe hepatic impairment because asenapine exposures were on average seven times higher than for patients with normal hepatic function. ${ }^{2}$ No dosage adjustment is required for patients with renal impairment. ${ }^{2}$ Asenapine can inhibit CYP2D6 and result in two-fold increases in paroxetine concentrations. ${ }^{26}$ Although not yet studied in a clinical trial, CYP2D6 inhibition may be of clinical importance when combing asenapine with CYP2D6-dependent antipsychotics, ${ }^{25}$ and the product labeling advises caution when coadministering asenapine with drugs that are both substrates and inhibitors for CYP2D6. ${ }^{2}$

Asenapine is the active moiety, and although there are approximately 38 metabolites, none are highly prevalent, and the metabolites have few relevant effects because of their lower affinity for the relevant receptors or because of their inability to cross the blood-brain barrier. ${ }^{25,26}$

Although the precise mechanism of action of asenapine in the treatment of schizophrenia is unknown, it is thought that antagonism at the dopamine D2 and serotonin 5HT2A receptors mediates antipsychotic activity. ${ }^{2}$ Asenapine has a unique receptor binding profile. ${ }^{30}$ The receptor binding profile in order of $\mathrm{Ki}$ values (in $\mathrm{nM}$ ) is: 5 -HT2C $(0.03)>5$-HT2A $(0.06)>5-H T 7(0.13)>5-H T 2 B(0.16)>5-H T 6(0.25)>$ D3 $(0.42)>\mathrm{H} 1(1.0)>\mathrm{D} 4(1.1)>\mathrm{NE}$ alpha $1(1.2)=\mathrm{NE}$ alpha 2 (1.2) $>$ D2 (1.3) > D1 (1.4) > 5-HT5 (1.6) > 5-HTA (2.5) $>$ 5-HT1B (4.0) $>$ H2 (6.2) $>>$ muscarinic M1 (8128). 


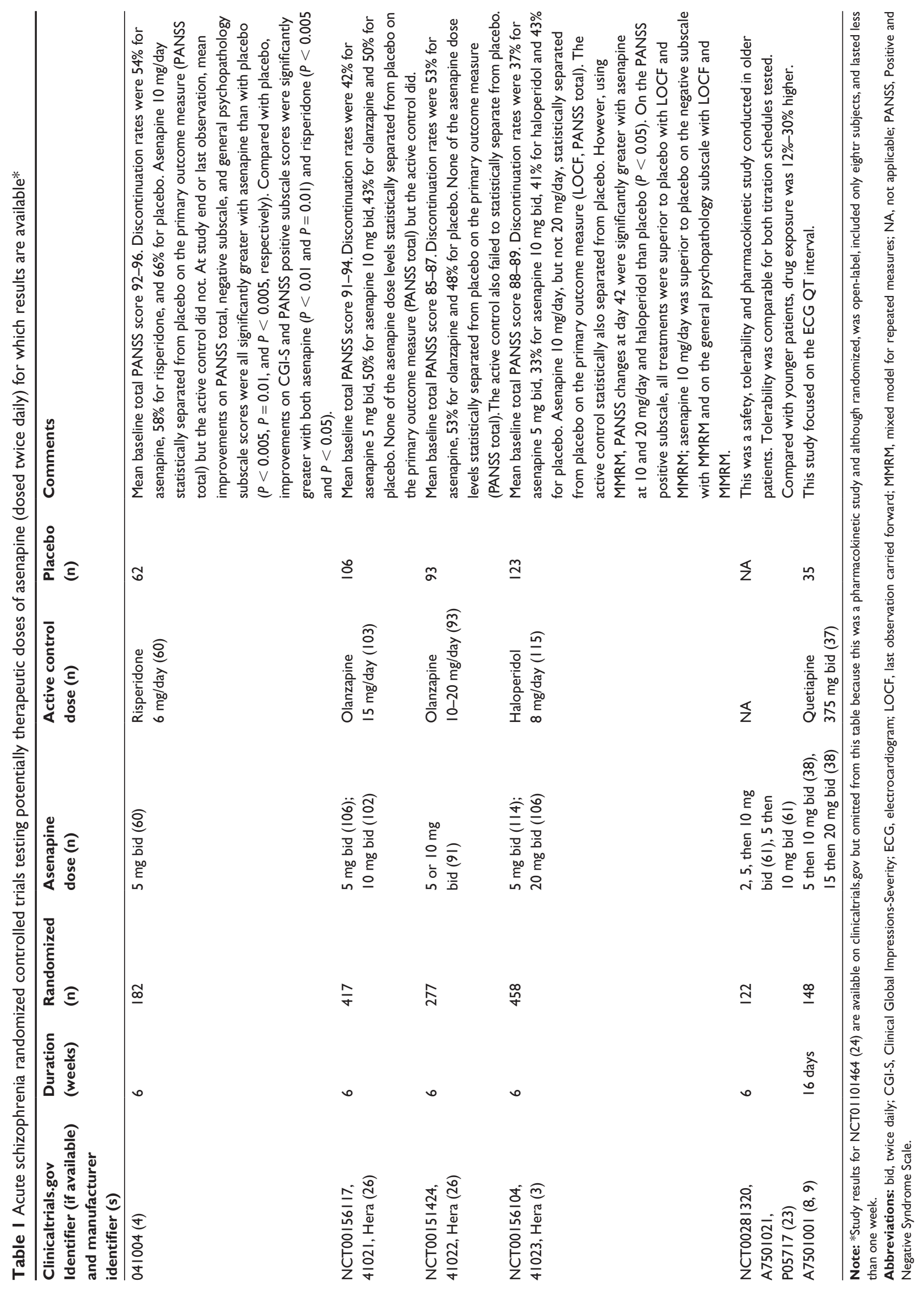




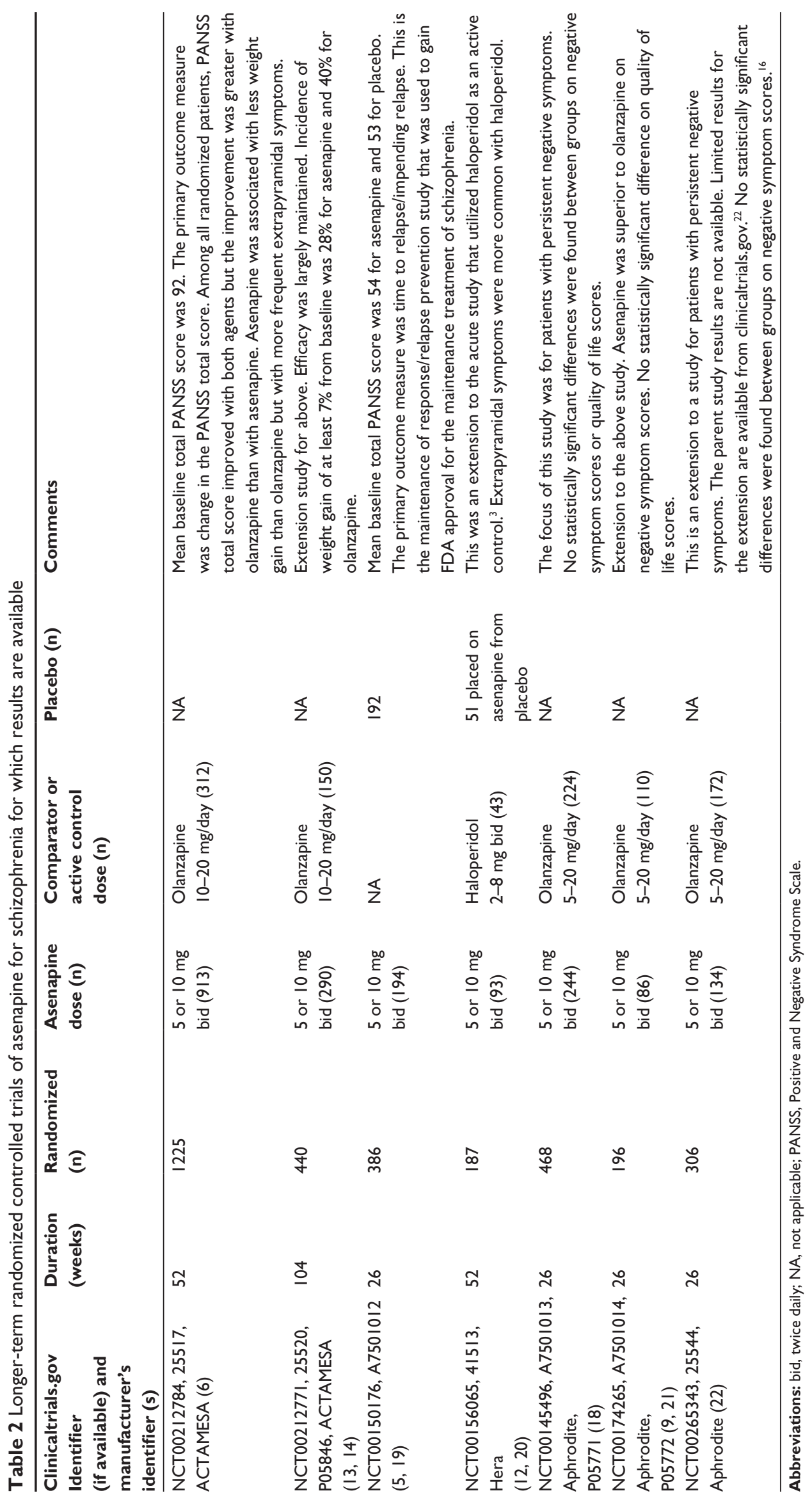


Asenapine is functionally an antagonist at the above receptors. The differing receptor affinity "fingerprints" of the available antipsychotics can potentially explain their heterogeneity in terms of their efficacy and tolerability profiles, and can explain in part the heterogeneity of antipsychotic response from patient to patient. ${ }^{31}$ It is noted that asenapine appears to have relatively higher potency at serotonin receptors than at dopamine receptors, including the serotonin HT7 receptor, which has been studied regarding object discrimination in rats, ${ }^{32}$ and may be a therapeutic target for achieving antidepressant and memory-enhancing actions. ${ }^{33}$ Receptor binding affinities may also help in predicting potential tolerability. For example, because of the noradrenergic alpha 1 receptor antagonism, asenapine can add to the effect of certain antihypertensive agents. ${ }^{2}$ Moreover, antagonism of histamine H1 receptors appears to be associated with the sedative effects of asenapine. ${ }^{26}$

As a caveat, receptor signatures are ordinarily quantified using cloned human receptors, and actual correlations with observed clinical effects needs to be assessed in people. Positron emission tomography can be used to do this, ${ }^{34}$ and asenapine has demonstrated dose-dependent dopamine D2 receptor occupancy (dose range $0.1-4.8 \mathrm{mg}$ ), with a significant correlation between D2 occupancy and plasma concentration. ${ }^{26}$ Sublingual administration of $4.8 \mathrm{mg}$ twice daily resulted in high levels of D2 occupancy, with a mean occupancy of 79\% at approximately 3-6 hours after dosing. ${ }^{26}$

\section{Efficacy in schizophrenia Short-term efficacy}

Short-term efficacy for schizophrenia was tested in four pivotal six-week, randomized, double-blind, placebo-controlled and active comparator-controlled multicenter studies that included asenapine doses of $5 \mathrm{mg}$ or $10 \mathrm{mg}$ twice daily (Table 1). Two studies were accepted by the FDA as supportive of asenapine's efficacy in the acute treatment of schizophrenia in adults. ${ }^{3,4}$ The other two studies consisted of one negative trial (study 41021; asenapine $5 \mathrm{mg}$ twice daily and $10 \mathrm{mg}$ twice daily did not statistically significantly separate from placebo on the primary outcome measure, but the active control, ie, olanzapine $15 \mathrm{mg} /$ day, did) and one failed trial (study 41022; asenapine flexibly dosed at $5 \mathrm{mg}$ or $10 \mathrm{mg}$ twice daily did not statistically significantly separate from placebo on the primary outcome measure and neither did the active control of olanzapine flexibly dosed at $10-20 \mathrm{mg} /$ day). ${ }^{26}$ Although these two latter unpublished trials could not be used to establish efficacy, the safety and tolerability outcomes were included in an integrated safety database.
In one of the positive trials, 458 patients with acute schizophrenia were randomly assigned to fixed-dose treatment with asenapine at $5 \mathrm{mg}$ twice daily, asenapine at $10 \mathrm{mg}$ twice daily (after one day at $5 \mathrm{mg}$ twice daily), placebo, or an active control for assay sensitivity, ie, haloperidol at $4 \mathrm{mg}$ twice daily. ${ }^{3}$ The study was international in scope and took place in 2005-2006. Patients were required to be hospitalized during the first two weeks of the study. The primary efficacy endpoint was change from baseline in the total score on the Positive and Negative Syndrome Scale (PANSS). There were two prespecified methodological approaches to assess efficacy, ie, analysis of covariance using last observation carried forward (LOCF) as the primary analysis and a mixed model for repeated measures (MMRM) as the secondary analysis. Discontinuation rates were $37.6 \%, 33.3 \%, 42.0 \%$, and $43.4 \%$ for asenapine $5 \mathrm{mg}$ twice daily, asenapine $10 \mathrm{mg}$ twice daily, haloperidol, and placebo groups, respectively. The discontinuation rates due to insufficient therapeutic response were $12.8 \%, 16.2 \%, 8.9 \%$, and $25.4 \%$, respectively, yielding a number needed to treat (number needed to treat $[\mathrm{NNT}]=35$, see Figure 1) versus placebo to avoid discontinuation because of an insufficient therapeutic response of 8,11, and 7 for asenapine $5 \mathrm{mg}$ twice daily, asenapine $10 \mathrm{mg}$ twice daily, and haloperidol, respectively. On both LOCF and MMRM analyses of change in PANSS total score, asenapine $5 \mathrm{mg}$ twice daily and haloperidol were both superior to placebo, with statistically significant differences seen from day 21 onwards. However, asenapine $10 \mathrm{mg}$ twice daily showed no advantage over placebo at any time point on the LOCF analysis and an advantage only at day 42 on the MMRM analysis, an effect that the authors suggest may have been due in part to the high placebo response evident in this trial. The secondary outcomes roughly mirrored the primary outcome. On the PANSS positive subscale score, asenapine $5 \mathrm{mg}$ twice daily and haloperidol were superior to placebo from day 21 onwards; asenapine $10 \mathrm{mg}$ twice daily showed an advantage at day 42 and study endpoint. On the LOCF analysis of change in the PANSS negative subscale, none of the treatments was superior to placebo. However, using MMRM, asenapine $5 \mathrm{mg}$ twice daily demonstrated an advantage at days 35 and 42. On both the LOCF and MMRM analyses of change in the PANSS general psychopathology subscale score, asenapine $5 \mathrm{mg}$ twice daily was superior to placebo from day 21 onwards, in contrast with haloperidol, which showed an advantage at day 21 only, and with asenapine $10 \mathrm{mg}$ twice daily, which showed no advantage at any time point. PANSS Marder factor scores were also determined, and on both the LOCF and MMRM analyses, all active 


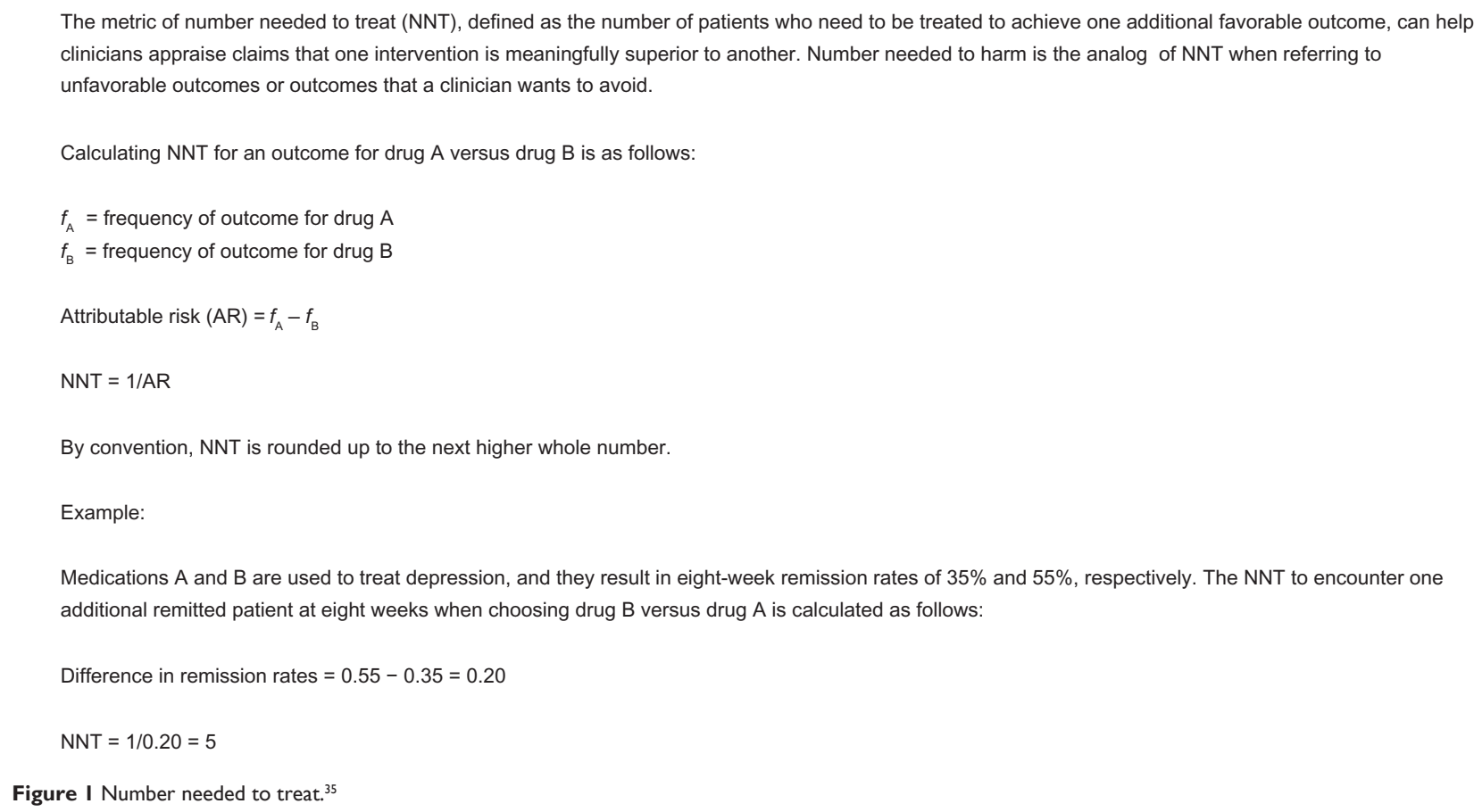

treatment regimens were superior to placebo on the positive factor, but none showed an advantage on the negative factor. On both analyses of the hostility/excitement factor, only haloperidol was superior to placebo. Asenapine $5 \mathrm{mg}$ twice daily was the only intervention that demonstrated superiority to placebo on the anxiety/depression factor (by MMRM) and the disorganized thought factor (by LOCF and MMRM). Categorical response, defined as a minimum decrease of $30 \%$ on the PANSS total score or a Clinical Global ImpressionImprovement (CGI-I) score of 1 (ie, very much improved) or 2 (much improved), were for PANSS 55\%, 49\%, 43\%, and $33 \%$, for asenapine $5 \mathrm{mg}$ twice daily, asenapine $10 \mathrm{mg}$ twice daily, haloperidol, or placebo, respectively, yielding a $\mathrm{NNT}=5,7$, and 10 versus placebo for asenapine $5 \mathrm{mg}$ twice daily, asenapine $10 \mathrm{mg}$ twice daily, and haloperidol, respectively. Similarly, CGI-I responders at endpoint were $48 \%, 44 \%, 44 \%$, and $34 \%$, for asenapine $5 \mathrm{mg}$ twice daily, asenapine $10 \mathrm{mg}$ twice daily, haloperidol or placebo, respectively, yielding a NNT $=8,10$, and 10 versus placebo for asenapine $5 \mathrm{mg}$ twice daily, asenapine $10 \mathrm{mg}$ twice daily, and haloperidol, respectively. Using change in CGI-I change as the outcome of interest, asenapine $5 \mathrm{mg}$ twice daily and haloperidol were both significantly superior to placebo from day 21 onwards (by LOCF and MMRM).

In the second short-term acute schizophrenia trial that was considered positive and supportive of asenapine, 182 patients were randomized to asenapine $5 \mathrm{mg}$ twice daily (titrated as $1 \mathrm{mg}$ twice daily on day $1,2 \mathrm{mg}$ twice daily on day 2, $3 \mathrm{mg}$ on day 3, $4 \mathrm{mg}$ twice daily on day 4, and $5 \mathrm{mg}$ on day 5), placebo, or an active control for assay sensitivity, ie, risperidone $3 \mathrm{mg}$ twice daily (titrated as $1 \mathrm{mg}$ twice daily on day 1, $2 \mathrm{mg}$ twice daily on day 2 , and $3 \mathrm{mg}$ twice daily on day 3). ${ }^{4}$ The study took place in the US in 2001-2002. Patients were required to be hospitalized during the first three weeks of the study. The primary efficacy endpoint was change from baseline on the PANSS total score, and was analyzed using LOCF. Discontinuation rates were $54.2 \%, 57.6 \%$, and $66.1 \%$ for asenapine, risperidone, and placebo groups, respectively. The discontinuation rates due to lack of efficacy were $15.2 \%$, $27.1 \%$, and $29.0 \%$, respectively, yielding a NNT $=8$ versus placebo to avoid a discontinuation due to lack of efficacy for asenapine and 53 for risperidone. Compared with placebo, asenapine produced significantly greater decreases in PANSS total scores from week 2 onwards. Risperidone did not separate statistically from placebo. Compared with placebo, asenapine and risperidone were associated with significantly greater decreases in Clinical Global Impression-Severity (CGI-S) scores from week 4 onwards. On the PANSS positive subscale, scores with asenapine demonstrated statistically greater improvement over placebo for asenapine from week 3 onwards, and with risperidone at weeks 1, 3, 5, and 6. On the PANSS negative and general psychopathology subscales at endpoint, asenapine demonstrated statistically superior improvement compared with placebo but risperidone did not. A post hoc MMRM analysis confirmed the overall efficacy of asenapine in the primary outcome measure. The authors 
speculated on the reasons why risperidone may not have performed as expected, including issues regarding dosing at $6 \mathrm{mg} /$ day. Not mentioned in the study report, but available elsewhere, ${ }^{26}$ are the categorical response rates. Using the criterion of a PANSS score reduction in at least $30 \%, 38 \%$ of the patients in the asenapine group were responders compared with $39 \%$ in the risperidone group and $25 \%$ in the placebo group, yielding a NNT $=8$ versus placebo for asenapine and $\mathrm{NNT}=7$ for risperidone. ${ }^{25}$

\section{Longer-term efficacy}

Longer-term studies are also available (Table 2). Published is a one-year double-blind study in 1225 patients with schizophrenia or schizoaffective disorder who were randomized to asenapine (5 mg twice daily for the first week and then flexible dosing of $5 \mathrm{mg}$ or $10 \mathrm{mg}$ twice daily) or olanzapine $(10 \mathrm{mg} /$ day for the first week and then flexible dosing of $10 \mathrm{mg}$ /day or $20 \mathrm{mg} /$ day). ${ }^{6}$ There was no placebo arm. The study was international in scope and was conducted in 2003-2006. Both inpatients and outpatients were eligible to participate. The primary outcome measure as noted in the clinicaltrials.gov record was change in total PANSS score at endpoint. ${ }^{36}$ Discontinuation rates were $61.4 \%$ for asenapine and $42.8 \%$ for olanzapine. The discontinuation rates because of insufficient therapeutic effect were $25.1 \%$ for asenapine and $14.5 \%$ for olanzapine (NNT $=10$ for olanzapine versus asenapine to avoid discontinuation because of insufficient therapeutic effect). In the LOCF analysis, changes from baseline in PANSS total score with asenapine and olanzapine were similar at week 6 , but showed a statistically significant difference in favor of olanzapine at endpoint. Among the patients who completed the year-long trial, changes in PANSS total score with asenapine and olanzapine were similar at week 6 and also at week 52. Categorical outcomes were provided for CGI-I scores; $66 \%$ of patients treated with olanzapine had an endpoint CGI-I of 1 or 2, compared with $52 \%$ for asenapine $(\mathrm{NNT}=8)$.

Efficacy for the maintenance phase of schizophrenia was demonstrated in a published 26-week placebo-controlled, double-blind, multicenter, clinical trial. ${ }^{5}$ Patients were randomized either to continue receiving asenapine or to receive placebo after having achieved stability on asenapine during 26 weeks of open-label treatment immediately prior. The study was conducted in the US during 2005-2008. The primary outcome measure was time to relapse/impending relapse (as determined by the investigator using specific criteria as well as specific scores on the PANSS and the CGI-S scale). Of the 700 enrolled patients who were treated with open-label asenapine, 386 met the stability criteria and were randomized. Times to relapse/impending relapse and discontinuation for any reason were significantly longer with asenapine than with placebo. The incidence of relapse/ impending relapse was $12.1 \%$ for asenapine and $47.4 \%$ for placebo $(\mathrm{NNT}=3)$. Completion rates were $69.6 \%$ for asenapine and $37.5 \%$ for placebo $(\mathrm{NNT}=4)$. The most commonly used dose of asenapine was $10 \mathrm{mg}$ twice daily in both the open-label and double-blind phases.

Asenapine was assessed over 52 weeks in an extension to the acute study that used haloperidol as an active control. ${ }^{3}$ Although not yet published, some of the data are available. ${ }^{12,20}$ Patients receiving active medication during the six-week trial were maintained on the same fixed-dose regimen during week 1 of the extension, after which dosing was flexible (asenapine $5 \mathrm{mg}$ or $10 \mathrm{mg}$ twice daily, haloperidol $2-8 \mathrm{mg}$ twice daily). Patients who had received placebo were given blinded asenapine ( $5 \mathrm{mg}$ twice daily during week 1 , flexible dosing at $5 \mathrm{mg}$ or $10 \mathrm{mg}$ twice daily thereafter). Of 272 patients who completed the six-week trial, 187 entered the extension and 66 completed 58 weeks of treatment (placebo/ asenapine 20; asenapine 30; haloperidol 16). The primary outcome measure was loss of effect over time, as measured by loss of effect in subjects who had at least a $30 \%$ decrease from baseline in the PANSS score at the end of the original trial $^{3}$ preceding the long-term extension. Loss of effect was defined as at least a $30 \%$ increase in the total PANSS score from the start of the extension study, subjective worsening of schizophrenia/request for dose increase, CGI-S of at least 6 , or discontinuation for lack of efficacy. The numbers of subjects for this analysis were 30 for placebo/asenapine, 65 for asenapine, and 29 for haloperidol. Actual treatment failure rates were similar during the 52-week extension: $85 \%$ of asenapine patients and $90 \%$ of haloperidol patients demonstrated loss of treatment effect. The Kaplan-Meier estimate of loss of effect was 0.86 for placebo/asenapine, 0.88 for asenapine, and 0.94 for haloperidol. Median survival time was 57 days for placebo/asenapine, 31 days for asenapine, and 85 days for haloperidol. Mean changes in PANSS total score from baseline of the six-week trial (LOCF) for asenapine and haloperidol, respectively, were -23.7 versus -22.6 at baseline of the extension, -21.9 versus -26.5 at week 52 , and -21.8 versus -26.7 at study endpoint.

Persistent negative symptoms of schizophrenia were also studied in patients receiving asenapine. ${ }^{16,37}$ These unpublished studies consist of two identically designed randomized clinical trials where patients were randomized to receive flexible-dose asenapine ( $5 \mathrm{mg}$ twice daily in week 
1 and then 5 or $10 \mathrm{mg}$ twice daily) or olanzapine $(10 \mathrm{mg} /$ day in week 1 and then $5-20 \mathrm{mg} /$ day) for up to 26 weeks. ${ }^{18,38}$ Corresponding 26-week double-blind extension studies were available to the completers, during which subjects were maintained on the treatment regimen used at the end of each core study, with no rerandomization. ${ }^{21,22}$ The primary outcome measure was change from core study baseline on the 16-item Negative Symptom Assessment (NSA-16) scale total score, analyzed using MMRM. Treatment differences on this outcome were observed only in the 26-week extension of a 26-week core study conducted in the Western hemisphere, ${ }^{18,21}$ and was reported in a conference abstract. ${ }^{9}$ Completion rates for the entire 52 weeks were $66.3 \%$ for asenapine and $80.9 \%$ for olanzapine $(\mathrm{NNT}=7)$. At week 26 of the core study, NSA-16 total score changes with asenapine and olanzapine did not differ significantly, but at week 52, NSA-16 total score changes were significantly greater with asenapine $(-15.8)$ versus olanzapine $(-11.0)$. In another abstract, ${ }^{16}$ results from a post hoc pooled analysis of all four trials (ie, the two core trials and their respective extensions) were provided. A total of 949 participants were randomized to treatment in the two core studies (asenapine, 485; olanzapine, 464). Of the 613 participants (asenapine, 277; olanzapine, 336) who completed 26 weeks of treatment, 502 (asenapine, 220; olanzapine, 282) entered the 26-week extensions and 412 (asenapine, 170; olanzapine, 242) completed an additional 26 weeks of treatment. Discontinuation rates due to lack of therapeutic effect (defined as worsening of schizophrenia as an adverse event plus lack of efficacy) was significantly greater for asenapine versus olanzapine for the first 26 weeks among all treated participants entering the core studies ( $13.6 \%$ versus $7.3 \%$, $\mathrm{NNT}=16$ ), and among all subjects entering the extensions $(5.5 \%$ versus $2.1 \%, \mathrm{NNT}=30)$. After 26 weeks of treatment, change from core study baseline in NSA-16 total score did not significantly differ between asenapine and olanzapine, but at 52 weeks the change from core study baseline in NSA-16 total score was significantly greater with asenapine than for olanzapine.

\section{Meta-analysis}

Presented at a conference was a meta-analysis, ${ }^{17}$ where data from all randomized head-to-head comparisons of asenapine with second-generation antipsychotics and published randomized head-to-head comparisons of second-generation antipsychotics in the treatment of schizophrenia were analyzed. This permitted indirect comparisons. Change in PANSS total score at week 6 was significantly greater with asenapine than with placebo (LOCF, -3.7; MMRM -4.1). The efficacy of asenapine relative to placebo was comparable with that of the combined active controls used in the studies (LOCF -4.1; MMRM -4.6). Analysis of PANSS responder rates (defined by an at least $30 \%$ decrease from baseline) revealed a NNT $=11$ versus placebo and was comparable with responder rates of the combined active controls (NNT = 12). Estimated PANSS total score difference between asenapine and other second-generation antipsychotics ranged from 3.9 points better than ziprasidone to 2.9 points worse than olanzapine. Risperidone and amisulpride slightly outperformed asenapine (1.0 and 0.5 points, respectively) but asenapine performed better than aripiprazole (1.6 points), quetiapine (1.1 points), sertindole (1.0 points), and clozapine ( 0.3 points).

\section{Dosing}

The product labeling recommends asenapine $5 \mathrm{mg}$ twice daily as the starting and target dose when treating adult patients with acute schizophrenia, ${ }^{2}$ based on the pattern of results observed in the short-term clinical trials. ${ }^{25}$ In contrast, the most commonly used dose in the relapse/maintenance trial $^{6}$ for both the open-label stabilization period and the randomized phase was $10 \mathrm{mg}$ twice daily. In a modeling and simulation study, ${ }^{39}$ asenapine $5 \mathrm{mg}$ and $10 \mathrm{mg}$ twice daily doses had similar efficacy. The FDA has requested that a study be done that can identify the lowest effective dose of asenapine for the treatment of adults with schizophrenia (ie, a trial of a dose lower than $5 \mathrm{mg}$ twice daily, such as $2.5 \mathrm{mg}$ twice daily). ${ }^{25}$

\section{Safety and tolerability}

According to the product labeling, ${ }^{2}$ the most commonly encountered adverse events in patients with schizophrenia are listed as akathisia, oral hypoesthesia (numbness), and somnolence. Rates and number needed to harm (NNH) versus placebo are outlined in Table 3. Akathisia may be dose-related. The product labeling describes somnolence as usually transient, with the highest incidence reported during the first week of treatment. ${ }^{2}$ Rates for somnolence are higher in bipolar patients treated with asenapine, which may be reflective of the overall different doses of asenapine used in the treatment of manic or mixed episodes (usually $10 \mathrm{mg}$ twice daily), or the different sensitivity of patients with bipolar disorder to complain actively of somnolence. Although somnolence was frequently reported, somnolence/ sedation led to discontinuation in only a small proportion $(0.6 \%)$ of patients treated with asenapine. ${ }^{2}$ 
A caveat is that these rates are that of spontaneously reported events. Patients in clinical trials may have a different threshold for complaining about side effects than persons treated in real-world practice. Directly asking patients about adverse effects, such as oral hypoesthesia, may yield higher rates. In healthy male subjects from a Phase I study of asenapine $5 \mathrm{mg},{ }^{29}$ the most common adverse events were oral paresthesia (sublingual, 75.8\%; supralingual, 55.9\%; buccal, $45.7 \%)$ and somnolence $(81.8 \% ; 76.5 \% ; 68.6 \%)$.

In the short-term study of asenapine with haloperidol as the active control, ${ }^{3}$ adverse events occurred in $44 \%$ and $52 \%$, $57 \%$, and $41 \%$ of the asenapine $5 \mathrm{mg}$ and $10 \mathrm{mg}$ twice daily, haloperidol, and placebo groups, respectively. Extrapyramidal symptoms (in most cases rated as mild or moderate) reported as adverse events occurred in $15 \%$ and $18 \%, 34 \%$, and $10 \%$ of the asenapine $5 \mathrm{mg}$ and $10 \mathrm{mg}$ twice daily, haloperidol, and placebo groups, respectively ( $\mathrm{NNH}=6$ for haloperidol versus asenapine and $\mathrm{NNH}=7$ for asenapine $5 \mathrm{mg}$ and $10 \mathrm{mg}$ twice daily, respectively). During the study, antiparkinsonian medication was initiated in $17 \%, 19 \%, 12 \%$, and $43 \%$ of patients in the asenapine $5 \mathrm{mg}$ and $10 \mathrm{mg}$ twice daily, placebo, and haloperidol groups, respectively $(\mathrm{NNH}=4$ for haloperidol versus asenapine and $\mathrm{NNH}=5$ for asenapine $5 \mathrm{mg}$ and $10 \mathrm{mg}$ twice daily, respectively). For akathisia specifically, rates were $5 \%$ and $12 \%, 15 \%$, and $3 \%$ for the asenapine $5 \mathrm{mg}$ and $10 \mathrm{mg}$ twice daily, haloperidol, and placebo groups, respectively. Oral hypoesthesia was reported in $11 \%$ and $9 \%$ with asenapine $5 \mathrm{mg}$ and $10 \mathrm{mg}$ versus $2 \%$ with placebo and $0 \%$ with haloperidol. Somnolence was reported in $9 \%$ and $8 \%$ of patients on asenapine $5 \mathrm{mg}$ and $10 \mathrm{mg}$ twice daily versus less than $1 \%$ with placebo and $2 \%$ with haloperidol. Across all groups, no more than $5 \%$ of patients had clinically significant weight change. Mean changes from baseline in levels of lipids, fasting glucose, and liver enzymes were small

Table 3 Commonly encountered (incidence $\geq 5 \%$ and two-fold greater than placebo for at least one of the doses) spontaneously reported adverse events as reported in product labeling for the acute treatment of schizophrenia: percentage of patients reporting reaction and number needed to harm versus placebo*

\begin{tabular}{llllll}
\hline Adverse event & \multicolumn{3}{l}{ Asenapine studies } \\
\cline { 2 - 6 } & Placebo & \multicolumn{2}{l}{$\begin{array}{l}\text { Asenapine } \\
\text { 10 } \mathbf{~ m g / d a y ~}\end{array}$} & \multicolumn{2}{l}{ Asenapine } \\
& \multicolumn{2}{l}{ 20 $\mathbf{~ m} /$ day } \\
\cline { 2 - 6 } & Rate & Rate & NNH & Rate & NNH \\
\hline Somnolence & $7 \%$ & $15 \%$ & 13 & $13 \%$ & 17 \\
Akathisia & $3 \%$ & $4 \%$ & 100 & $11 \%$ & 13 \\
Oral hypoesthesia & $1 \%$ & $6 \%$ & 20 & $7 \%$ & 17 \\
\hline
\end{tabular}

Notes: *Data from Table 2 in product labeling for asenapine. ${ }^{2}$ in all treatment groups and comparable with those seen with placebo. No patients in any treatment group had abnormal postbaseline total cholesterol levels. The percentages of patients with abnormal postbaseline fasting glucose levels were $1 \%, 2 \%, 1 \%$, and $3 \%$ in the asenapine $5 \mathrm{mg}$ and $10 \mathrm{mg}$ twice daily, placebo, and haloperidol groups, respectively. From baseline to last assessment, prolactin levels decreased in the placebo group and both asenapine groups, but increased in the haloperidol group. The percentages of patients with abnormal postbaseline prolactin levels (greater than four times the upper limit of normal) were $4 \%, 5 \%, 2 \%$, and $10 \%$ in the asenapine $5 \mathrm{mg}$ and $10 \mathrm{mg}$ twice daily, placebo, and haloperidol groups, respectively. Incidence rates of clinically relevant weight gain, defined as at least a 7\% increase from baseline, were $5 \%, 4 \%, 2 \%$, and $4 \%$ in the asenapine $5 \mathrm{mg}$ and $10 \mathrm{mg}$ twice daily, placebo, and haloperidol groups, respectively.

In the short-term study of asenapine with risperidone as the active control, ${ }^{4}$ adverse events occurred in $83 \%, 79 \%$, and $90 \%$ of patients in the asenapine, placebo, and risperidone groups, respectively. The most frequently reported adverse events were insomnia, somnolence, nausea, anxiety, and agitation in the asenapine group; agitation, headache, anxiety, and dizziness in the placebo group; and insomnia, somnolence, anxiety, agitation, and headache in the risperidone group. Risperidone-treated patients were more likely to report symptoms resembling hypertonia ( $12 \%$ versus $0 \%$ for asenapine and $3 \%$ for placebo) and hyperkinesia (7\% versus $0 \%$ for asenapine and placebo) as adverse events, and more likely to use antiparkinsonian drugs $(17 \%, 21 \%$ and $31 \%$, for asenapine, placebo, and risperidone, respectively $(\mathrm{NNH}=8$ for risperidone versus asenapine). The incidence of clinically relevant weight gain was $17.0 \%$ with risperidone versus $4.3 \%$ with asenapine and $1.9 \%$ with placebo $(\mathrm{NNH}=8$ for risperidone versus asenapine). The proportion of patients with normal baseline prolactin levels but postbaseline levels at least twice the upper limit of normal was $79 \%$ for risperidone compared with $9 \%$ for asenapine and $2 \%$ for placebo $(\mathrm{NNH}=2$ for risperidone versus asenapine). Abnormal postbaseline fasting glucose levels occurred in $14 \%, 12 \%$, and $20 \%$ of patients treated with asenapine, placebo, and risperidone, respectively. At least one postbaseline QTc interval of at least 450 msec was observed in $9 \%, 10 \%$, and $18 \%$ of patients treated with asenapine, placebo, and risperidone, respectively, but there were no reports of QT interval prolongation greater than $500 \mathrm{msec}$ in any treatment group. Mean changes in QTc from baseline were $+4.6 \mathrm{msec}$ with asenapine, $-1.6 \mathrm{msec}$ with placebo, and $+4.4 \mathrm{msec}$ with risperidone. 
A specially designed thorough 16-day QT study has been published. ${ }^{78}$ Patients were randomized to asenapine $5 \mathrm{mg}$ twice daily followed by $10 \mathrm{mg}$ twice daily, asenapine $15 \mathrm{mg}$ twice daily followed by $20 \mathrm{mg}$ twice daily, quetiapine $375 \mathrm{mg}$ twice daily, or placebo. At maximum plasma concentration for all asenapine doses, the exposure-response model predicted a mean QTc increase of less than $5 \mathrm{msec}$. The model predicted a mean increase of $7-8 \mathrm{msec}$ for quetiapine. The corresponding upper bounds of the $95 \%$ confidence intervals were $7.5 \mathrm{msec}$ and $11.2 \mathrm{msec}$ for asenapine and quetiapine, respectively. Using another modeling technique, QTc estimates for asenapine were $7-10 \mathrm{msec}$.

Information about asenapine in elderly patients with psychosis is also available. ${ }^{10,11,23}$ An unpublished six-week randomized trial enrolled patients aged at least 65 years of age with psychotic symptoms (not related to dementia), defined as a PANSS score of at least 4 on at least one prespecified item (delusions, hallucinatory behavior, excitement, hostility, poor impulse control), a PANSS total score greater than 50, and a CGI-S score of at least 3. Asenapine was given in two treatment schedules, ie, two days at $2 \mathrm{mg}$ twice daily, two days at $5 \mathrm{mg}$ twice daily, and $10 \mathrm{mg}$ twice daily thereafter (2-5-10); or four days at $5 \mathrm{mg}$ twice daily and $10 \mathrm{mg}$ twice daily thereafter (5-10). Drug treatment was conducted under double-blind conditions during the initial six days, and was open-label for the remainder of the trial. Primary outcomes were related to occurrences of adverse events as well as pharmacokinetic variables..$^{23}$ The mean age of the subjects was 71 years. The completion rate was $62.3 \%$. Tolerability was comparable across treatment schedules. The percentage of subjects who experienced an adverse event was $72 \%$ in each group. The percentage of subjects who discontinued because of an adverse event was $20 \%$ in the $2-5-10$ titration group and $15 \%$ in the $5-10$ titration group. The rate of serious adverse events was $10 \%$ for the 2-5-10 titration group and 5\% for the 5-10 titration group. Adverse events reported by at least $5 \%$ of patients included hypertension $(8.2 \%)$, headache $(6.6 \%)$, and somnolence $(6.6 \%)$, and the incidence of extrapyramidal-related adverse events was $5.7 \%$. Mean weight change at endpoint was $0.4 \mathrm{~kg}$; clinically significant weight gain was reported in two patients (1.6\%).

Data about the longer-term tolerability of asenapine is available from the longer-term studies. In the relapse/ maintenance study, ${ }^{5}$ the most frequently reported adverse events with asenapine versus placebo during the double-blind phase were anxiety $(8.2 \%$ versus $10.9 \%)$, increased weight (6.7\% versus $3.6 \%)$, and insomnia (6.2\% versus $13.5 \%)$. Anxiety and insomnia after being switched to placebo from asenapine can be logically anticipated because patients were required to demonstrate stability on asenapine prior to randomization. The incidence of clinically relevant weight gain was $3.7 \%$ with asenapine versus $0.5 \%$ with placebo. Mean changes in scales measuring extrapyramidal symptoms were similar with asenapine and placebo.

Of greater clinical relevance than long-term comparisons of asenapine with placebo are comparisons of asenapine with other antipsychotics. In a one-year, double-blind, randomized controlled trial comparing asenapine with olanzapine, ${ }^{6}$ the incidence of treatment-emergent adverse events was $82 \%$ in both groups. Most reported adverse events were rated as mild or moderate. For the asenapine and olanzapine groups, respectively, incidence rates of treatment-emergent serious adverse events were $19 \%$ and $12 \%(\mathrm{NNH}=15)$, and rates of treatment-related serious adverse events were $6 \%$ and $2 \%$ $(\mathrm{NNH}=25)$. The mortality rate was less than $1 \%$ in both groups, and none were considered treatment-related per se; there were seven deaths in the asenapine group (five by suicide, among 11 attempts) and one death in the olanzapine group (by suicide, among six attempts). Mean weight gain was $0.9 \mathrm{~kg}$ with asenapine and $4.2 \mathrm{~kg}$ with olanzapine. The proportion of patients experiencing clinically relevant weight gain was approximately $35 \%$ for olanzapine and approximately $15 \%$ for asenapine $(\mathrm{NNH}=5)$. No notable changes or between-group differences were seen in measures of total cholesterol or glucose, but triglyceride levels rose substantially with olanzapine and declined slightly with asenapine. Extrapyramidal symptoms reported as adverse events were more common with asenapine (18\%) compared with olanzapine $(8 \%, \mathrm{NNH}=10)$. The most commonly reported type of movement disorder in the asenapine and olanzapine groups was akathisia, with treatment-emergent rates of $10 \%$ for asenapine and $4 \%$ for olanzapine $(\mathrm{NNH}=17)$. Extrapyramidal symptoms, most commonly akathisia, were the reported cause of premature discontinuation in 12 patients taking asenapine $(1 \%)$ and three patients taking olanzapine (1\%). Anticholinergic drug use among all treated patients at endpoint was $6 \%$ for asenapine and $2 \%$ for olanzapine $(\mathrm{NNH}=25)$. On formal assessment of extrapyramidal symptoms, mean scores on rating scales decreased from baseline in both groups. Markedly abnormal increases (to levels greater than three times the upper limit of normal) in alanine transaminase were seen in 3\% of the asenapine group and $11 \%$ of the olanzapine group $(\mathrm{NNH}=13)$; for aspartate transaminase, the percentages were $1 \%$ and $3 \%$, respectively $(\mathrm{NNH}=50)$. The incidence of abnormalities in vital signs was low and not different between the groups. 
Electrocardiographic abnormalities, mostly QT prolongation, were observed in $2.4 \%$ and $1.3 \%$ of patients in the asenapine and olanzapine groups, respectively $(\mathrm{NNH}=91)$; however, there were no instances of QTc of $\geq 500 \mathrm{msec}$ at any time during treatment.

An unpublished two-year blinded extension to the above study was also completed, providing three years of data. ${ }^{13,14}$ Of the 440 enrolled patients, 114 discontinued; rates of discontinuation were $30 \%$ for asenapine and $18 \%$ for olanzapine $(\mathrm{NNT}=9)$. The most common reasons for discontinuation were withdrawal of consent (asenapine, $11.0 \%$; olanzapine, $12.7 \%$ ) and treatment-emergent adverse events (asenapine, 10.3\%; olanzapine, 2.0\%). Mean weight gain from core study baseline was $1.6 \mathrm{~kg}$ with asenapine and $5.0 \mathrm{~kg}$ with olanzapine, and the incidence of clinically relevant weight gain was $28 \%$ and $40 \%$, respectively $(\mathrm{NNH}=9)$. The incidence of extrapyramidal symptom adverse events during the entire treatment period was $20 \%$ with asenapine and $11 \%$ with olanzapine $(\mathrm{NNH}=12)$, and during the extension alone was $4.5 \%$ and $3.3 \%$, respectively $(\mathrm{NNH}=84)$.

Safety and tolerability data are available from the 52-week extension to the acute study that used haloperidol as an active control. ${ }^{12,20}$ From the baseline of the six-week trial to the end of the extension, incidence rates of treatmentemergent adverse events were $88 \%, 85 \%$, and $86 \%$ for placebo/asenapine, asenapine, and haloperidol, respectively. The most common treatment-emergent adverse events not related to worsening of schizophrenia in the placebo/ asenapine, asenapine, and haloperidol groups were insomnia (38\%, 32\%, 21\%), parkinsonism (10\%, 17\%, 28\%), akathisia $(10 \%, 16 \%, 28 \%)$, and headache $(26 \%, 16 \%, 19 \%)$. The incidence of clinically relevant weight gain was $13 \%$, $19 \%$, and 15\%. Extrapyramidal symptom adverse events was higher with haloperidol (54\%) than with placebo/ asenapine $(18 \%)$ or asenapine $(35 \%)(\mathrm{NNH}=6$ for haloperidol versus asenapine). Mean changes in prolactin levels were $-10.8,-13.8$, and $-23.7 \mu \mathrm{g} / \mathrm{L}$ for placebo/asenapine, haloperidol, and asenapine, respectively. The incidence of markedly abnormal results on fasting glucose, hemoglobin A1c, cholesterol, and triglycerides was low, with no notable between-group differences. Two deaths occurred (one each with asenapine and haloperidol), and neither was considered treatment-related.

Safety information is available from the studies examining negative symptoms in schizophrenia but these studies have not yet been published and thus their results have only been partially disclosed. In one of the extension studies that was presented at a meeting, ${ }^{9}$ the incidence of treatmentemergent adverse events was $82 \%$ with asenapine and $91 \%$ with olanzapine $(\mathrm{NNH}=12)$. The incidence of extrapyramidal adverse events was $24 \%$ for asenapine and $10 \%$ for olanzapine $(\mathrm{NNH}=8)$. Weight changes from core study baseline to week 52 were $1.2 \mathrm{~kg}$ for asenapine and $2.2 \mathrm{~kg}$ for olanzapine.

A pooled analysis of weight change and metabolic effects of asenapine has also been presented. ${ }^{15}$ A database of 1748 patients who participated in trials that included a placebo control (989 receiving asenapine and 759 receiving placebo) and 3430 patients who participated in trials that included an olanzapine control (2067 receiving asenapine and 1363 receiving olanzapine) were included among 13 schizophrenia and four bipolar disorder trials, short-term and long-term, including extensions. Of note, four trials (two in patients with schizophrenia and two in patients with bipolar disorder) included both placebo controls and olanzapine controls. In 15 of the 17 trials, asenapine doses were $5 \mathrm{mg}$ or $10 \mathrm{mg}$ twice daily. Olanzapine doses were 5-20 mg/day. For the placebo-controlled trials, asenapine exposure was at a mean dose of $16.2 \mathrm{mg}$ /day with a mean treatment duration of 25.1 days. Mean exposure to placebo was for 24.8 days. For the olanzapine-controlled studies, asenapine exposure was at a mean dose of $15.3 \mathrm{mg} /$ day with a mean treatment duration of 207.3 days. Olanzapine exposure was at a mean dose of $15.0 \mathrm{mg}$ /day with a mean treatment duration of 226.2 days. At endpoint, the mean weight change with asenapine was significantly greater than with placebo (1.2 kg versus $0.1 \mathrm{~kg})$ and significantly less than with olanzapine $(0.9 \mathrm{~kg}$ versus $3.1 \mathrm{~kg})$. Mean change in total cholesterol, low-density lipoprotein, and high-density lipoprotein at endpoint was not significantly different between asenapine and placebo. Mean change in triglycerides differed significantly for asenapine versus placebo $(1.8 \mathrm{mg} / \mathrm{dL}$ versus $-12.2 \mathrm{mg} / \mathrm{dL})$. Percentages of patients with clinically relevant lipid changes in categorical analyses tended to be higher with asenapine than with placebo, with the exception of low-density lipoprotein, for which no differences were seen. Mean change was significantly lower with asenapine than with olanzapine for total cholesterol $(-0.4 \mathrm{mg} / \mathrm{dL}$ versus $6.2 \mathrm{mg} / \mathrm{dL})$, low-density lipoprotein $(-0.3 \mathrm{mg} / \mathrm{dL}$ versus $3.1 \mathrm{mg} / \mathrm{dL})$, and fasting triglycerides $(-0.9 \mathrm{mg} / \mathrm{dL}$ versus $24.3 \mathrm{mg} / \mathrm{dL})$. Mean change in high-density lipoprotein was significantly greater with asenapine than olanzapine $(1.3 \mathrm{mg} / \mathrm{dL}$ versus $-0.2 \mathrm{mg} / \mathrm{dL}$ ). At endpoint, mean change in fasting glucose was significantly higher for asenapine versus 
placebo $(1.9 \mathrm{mg} / \mathrm{dL}$ versus $-1.6 \mathrm{mg} / \mathrm{dL})$ but was numerically lower for asenapine versus olanzapine (2.0 versus $3.3 \mathrm{mg} / \mathrm{dL}$ ). Although the abstract states that categorical outcomes when comparing asenapine versus placebo generally reflect the mean changes in the variables mentioned, the actual values are not provided.

\section{Other outcomes}

The controlled clinical trials available provide limited information regarding potential changes in outcomes other than psychotic symptoms. In one of the short-term trials, secondary outcomes included the Calgary Depression Scale for Schizophrenia (CDSS), the Modified International Suicide Prevention Trial (InterSePT) Scale for Suicidal Thinking, and the Readiness to Discharge Questionnaire (RDQ). ${ }^{3}$ On LOCF analysis of change in CDSS score, significant improvement was seen with asenapine at $5 \mathrm{mg}$ twice daily starting at day 21; no statistically significant advantage over placebo was observed with asenapine $10 \mathrm{mg}$ twice daily or haloperidol. On the Modified InterSePT Scale for Suicidal Thinking and the RDQ, changes from baseline to endpoint were small in all groups, and no active treatment showed a significant difference from placebo. In a Kaplan-Meier analysis, $50 \%$ of patients were ready for discharge on day 15 with asenapine $5 \mathrm{mg}$ twice daily and haloperidol, on day 16 with asenapine $10 \mathrm{mg}$ twice daily, and on day 20 for placebo.

In the one-year study comparing asenapine with olanzapine, ${ }^{6}$ increases in mean Subjective Well-being under Neuroleptic treatment scale scores were observed for both asenapine and olanzapine. However, no relevant change from baseline was observed on either the mental or physical component of the 12-item Short Form scale, suggesting to the authors that patients' overall health status was not notably affected by treatment with either asenapine or olanzapine. Moreover, there were no notable changes within groups or significant between-group differences in living situation, employment, or level of functioning. Among the outpatient subjects in the trial, hospitalization occurred in $6 \%$ of those on asenapine and $3 \%$ in those on olanzapine $(\mathrm{NNH}=34)$. However, the total number of hospital days during the trial was marginally lower for asenapine than for olanzapine (mean 34.9 days and 36.3 days, respectively). In terms of preferences and ranking, at study end, $34 \%$ of asenapine-treated patients and $37 \%$ of investigators considered asenapine much better than previous antipsychotic medication; corresponding percentages for olanzapine were $40 \%$ and $48 \%$, respectively. Quality of life scale changes was recorded in the extension to the Western hemisphere study comparing asenapine with olanzapine for patients with predominantly negative symptoms; ${ }^{9}$ improvement was noted in both treatment arms, with no relevant between-group differences, as was also observed in the parent study. ${ }^{18}$

The market uptake of asenapine was reported in a brief paper. ${ }^{40}$ Postlaunch retail prescription trends of asenapine were assessed. In the 12 months since launch, asenapine gained a $0.22 \%$ share of the second-generation antipsychotic market. The share of new patient prescriptions was $0.44 \%$; this is double the total market share and may be a predictor of future growth. About $77 \%$ of total prescriptions of asenapine were generated by psychiatrists; this is higher than that for other second-generation antipsychotics. From the data reported in the study, it is unknown what proportion of asenapine prescriptions were for patients with schizophrenia versus those with bipolar disorder.

\section{Conclusion}

Selecting the "right" antipsychotic for the "right" patient is complex, and can involve trial and error. Factors in treatment selection include prior history of therapeutic response, prior history of tolerability with other agents, and individual patient values and preferences regarding both the symptoms to be targeted and the side effects to be avoided. Adherence can be the biggest obstacle to success; lack of consistent adherence is not uncommon in patients with schizophrenia, who may not perceive themselves as being ill or otherwise see no value in the medications being offered. Nonetheless, given the considerable heterogeneity in antipsychotic efficacy and tolerability among the available choices, as well as large individual variation in treatment response, having different options in order to optimize efficacy and tolerability for the individual patient is definitely desirable. ${ }^{1}$

Asenapine's efficacy in the treatment of schizophrenia is evidenced in both short-term acute clinical trials and in longer-term studies. Although a dose of $5 \mathrm{mg}$ twice daily was found to be efficacious in the acute trials, the 26-week maintenance trial was flexibly dosed, and the modal dose of asenapine was $10 \mathrm{mg}$ twice daily both in the open-label stabilization phase and in the randomized phase. In the head-to-head 52-week study comparing asenapine with olanzapine, changes from baseline in PANSS total score with asenapine and olanzapine were similar at week 6 , but showed a statistically significant difference in favor of olanzapine at 
endpoint (by LOCF). For observed cases (ie, patients who completed the entire study) changes in PANSS total score with asenapine and olanzapine were similar at week 6 and also at week 52 .

In the mind of the clinician, comparisons of asenapine will likely be made against other "metabolically-friendly" second-generation antipsychotics, such as ziprasidone, aripiprazole, iloperidone, and lurasidone. ${ }^{41}$ Tradeoffs among the choices include issues such as once-daily versus twice-daily dosing, the need for dose titration, special requirements for administration with or without food, as well as specific side effect profiles (see also Table 4 in a paper by the author ${ }^{41}$ and Table 4 in another paper by the author ${ }^{42}$ ). Relative efficacy rankings among these five agents (asenapine, ziprasidone, aripiprazole, iloperidone, and lurasidone) are not known, and will require specifically designed and adequately powered head-to-head studies. Head-to-head comparisons with the older second-generation antipsychotics, such as quetiapine and risperidone, would also be of interest, as well as an expansion of work already being done comparing asenapine with olanzapine. In the meantime, meta-analyses can be performed that can indirectly estimate potential differences, ${ }^{17}$ as has been done with the older secondgeneration antipsychotics. ${ }^{43-45}$

Available so far are the results of carefully conducted controlled trials that enrolled patients who fulfilled strict inclusion and exclusion criteria. All studies have been conducted by its manufacturer. The different studies for asenapine that examined acute use, maintenance use, and negative symptoms targeted somewhat different populations. These research subjects can be dissimilar to patients commonly treated in a routine clinical environment, particularly in terms of severity of illness, physical and psychiatric comorbidities (such as substance use), concomitant use of other antipsychotic medications, or dangerous behaviors. Known nonresponders to antipsychotics and patients recently treated with clozapine are commonly also excluded from participation in registration trials. There is a clear need for pragmatic clinical trials with more liberal enrollment criteria (permitting greater generalizability) that can help place antipsychotics such as asenapine into clinical perspective. Also desirable would be specific studies to ascertain the efficacy and tolerability profiles of asenapine in patients during their first episode of schizophrenia.

Asenapine remains unique as being the only antipsychotic that is absorbed in the oral mucosa. This renders impossible the surreptitious "cheeking" that can occur among patients who have an ambivalent attitude about adherence. Although asenapine is reasonably well tolerated, the possibility of oral hypoesthesia may be a new event for the patient, necessitating advance warning that this can occur. In the clinical trial program itself, oral hypoesthesia rarely led to discontinuation from the clinical trial (observed in $7 / 2251$ or $0.3 \%$ for asenapine versus $0 \%$ for all other groups). ${ }^{26}$ Dysgeusia (distorted or bad taste), has largely been alleviated with a black cherry-flavored formulation of asenapine.

In summary, the place of asenapine in the treatment of schizophrenia is likely to be for patients in whom metabolic concerns are important, and for patients who would prefer a sublingual preparation. Specific obstacles to the first-line use of asenapine are the recommendations for twice-daily versus once-daily administration and the recommendation to avoid food or liquids for 10 minutes after dosing. Cost may be a further impediment, given the availability of inexpensive generic versions of risperidone in the US, as well as other generic second-generation antipsychotics in other countries.

\section{Disclosure}

LC is a consultant for, has received honoraria from, or has conducted clinical research supported by the following companies: Abbott Laboratories, AstraZeneca Pharmaceuticals, Avanir Pharmaceuticals, Azur Pharma Inc, Barr Laboratories, Bristol-Myers Squibb, Eli Lilly and Company, Forest Research Institute, GlaxoSmithKline, Janssen Pharmaceuticals, Jazz Pharmaceuticals, Merck, Novartis, Noven Pharmaceuticals, Pfizer Inc, Shire, Sunovion, Valeant Pharmaceuticals, and Vanda Pharmaceuticals.

\section{References}

1. Volavka J, Citrome L. Oral antipsychotics for the treatment of schizophrenia: Heterogeneity in efficacy and tolerability should drive decision-making. Expert Opin Pharmacother. 2009;10(12):1917-1928.

2. Saphris (asenapine) sublingual tablets. Merck and Company Inc. Product labelling, revised January 2011. Available from: http:/www.spfiles.com/ pisaphrisv1.pdf. Accessed April 6, 2011.

3. Kane JM, Cohen M, Zhao J, Alphs L, Panagides J. Efficacy and safety of asenapine in a placebo- and haloperidol-controlled trial in patients with acute exacerbation of schizophrenia. J Clin Psychopharmacol. 2010; 30(2):106-115.

4. Potkin SG, Cohen M, Panagides J. Efficacy and tolerability of asenapine in acute schizophrenia: A placebo- and risperidone-controlled trial. J Clin Psychiatry. 2007;68(10):1492-1500.

5. Kane JM, Mackle M, Snow-Adami L, Zhao J, Szegedi A, Panagides J. A randomized placebo-controlled trial of asenapine for the prevention of relapse of schizophrenia after long-term treatment. J Clin Psychiatry. 2011;72(3):349-355.

6. Schoemaker J, Naber D, Vrijland P, Panagides J, Emsley R. Long-term assessment of asenapine vs. olanzapine in patients with schizophrenia or schizoaffective disorder. Pharmacopsychiatry. 2010;43(4):138-146. 
7. Chapel S, Hutmacher MM, Haig G, et al. Exposure-response analysis in patients with schizophrenia to assess the effect of asenapine on QTc prolongation. J Clin Pharmacol. 2009;49(11):1297-1308.

8. Chapel S, Hutmacher MM, Bockbrader H, de Greef R, Lalonde RL. Comparison of QTc data analysis methods recommended by the ICH E14 guidance and exposure-response analysis: Case study of a thorough QT study of asenapine. Clin Pharmacol Ther. 2011;89(1): $75-80$.

9. Cazorla P, Panagides J, Zhao J, et al. Long-term efficacy of asenapine in people with persistent negative symptoms of schizophrenia. Int $J$ Neuropsychopharmacol. 2010;13 Suppl 1:215.

10. Dubovsky SL, Frobose C, Phiri P, Panagides J. Short-term tolerability, safety, and pharmacokinetic profile of asenapine in older patients with psychosis. Schizophr Res. 2010;117(2-3):263-264.

11. Dubovsky S, Frobose C, Phiri P, Panagides J. Asenapine: Short-term tolerability, safety, and pharmacokinetic profile in older patients with psychosis. Eur Neuropsychopharmacol. 2010;20 Suppl 3: S489-S490.

12. Meltzer H, Cohen M, Snow-Adami L, et al. Long-term safety and maintenance of effect of asenapine in patients with acute exacerbation of schizophrenia. Eur Neuropsychopharmacol. 2009;19 Suppl 3: S536-S537.

13. Schoemaker J, Naber D, Jansen W, Panagides J, Emsley R. Safety and efficacy of long-term asenapine versus olanzapine in schizophrenia or schizoaffective disorder patients. Eur Psychiatry. 2010;25 Suppl 1: 1621.

14. Schoemaker J, Stet L, Naber D, Panagides J, Emsley R. Safety and efficacy of long-term asenapine versus olanzapine in schizophrenia or schizoaffective disorder patients. Int J Neuropsychopharmacol. 2010; 13 Suppl 1:238.

15. Zhao J, Van Hoek P, Cazorla P, et al. Weight change and metabolic effects of asenapine in placebo- or olanzapine-controlled studies. Neuropsychopharmacology. 2010;35 Suppl 1:S322.

16. Potkin P, Phiri P, Zhao J, et al. A pooled analysis of the effects of asenapine on the persistent negative symptoms of schizophrenia. Neuropsychopharmacology. 2010;35 Suppl 1:S213.

17. Szegedi A, Verweij P, Van Duijnhoven W, et al. Efficacy of asenapine for schizophrenia: Comparison with placebo and comparative efficacy of all atypical antipsychotics using all available head-to-head randomized trials using meta-analytical techniques. Neuropsychopharmacology. 2010;35 Suppl 1:S105.

18. Schering-Plough. Efficacy and safety of asenapine compared with olanzapine in patients with persistent negative symptoms of schizophrenia. Study NCT00145496. Available from: http:// www.clinicaltrials.gov/ct2/show/record/NCT00145496. Accessed April 6, 2011.

19. Schering-Plough. To determine long term efficacy and safety of asenapine in schizophrenic patient population. Study NCT00150176. Available from: http://www.clinicaltrials.gov/ct2/show/record/NCT00150176. Accessed April 6, 2011.

20. Schering-Plough. Long-term efficacy and safety of asenapine using haloperidol as a positive control. Study NCT00156065. Available from: http://www.clinicaltrials.gov/ct2/show/record/NCT00156065. Accessed April 6, 2011.

21. Schering-Plough. 6-month extension trial of asenapine with olanzapine in negative symptom patients who completed the first 6-month trial. Study NCT00174265. Available from: http://www.clinicaltrials. gov/ct2/show/record/NCT00174265. Accessed April 6, 2011.

22. Schering-Plough. 6-month extension trial of asenapine with olanzapine in negative symptom patients who completed the protocol 25543. Study NCT00265343. Available from: http://www.clinicaltrials.gov/ ct2/show/record/NCT00265343. Accessed April 6, 2011.

23. Schering-Plough. Study of asenapine in elderly subjects with psychosis. Study NCT00281320. Available from: http://www.clinicaltrials.gov/ct2/ show/record/NCT00281320. Accessed April 6, 2011.
24. Schering-Plough. A trial to determine whether two differing strength tablets $(3 \times 5 \mathrm{mg}$ versus $1 \times 15 \mathrm{mg})$ of sublingually Org 5222 (asenapine) are safe and equal in subjects with schizophrenia or schizoaffective disorder. Study NCT01101464. Available from: http:// www.clinicaltrials.gov/ct2/show/record/NCT01101464. Accessed April 6, 2011

25. Citrome L. Asenapine for schizophrenia and bipolar disorder: A review of the efficacy and safety profile for this newly approved sublingually absorbed second-generation antipsychotic. Int J Clin Pract. 2009; 63(12):1762-1784.

26. United States Food and Drug Administration. Saphris (asenapine) sublingual tablets. Briefing book. July 30, 2009. Available from: http:// www.fda.gov/downloads/AdvisoryCommittees/CommitteesMeetingMaterials/Drugs/PsychopharmacologicDrugsAdvisoryCommittee/ UCM173877.pdf. Accessed April 6, 2011.

27. Schering-Plough Research Institute. Saphris (asenapine) sublingual tablets. Briefing document (background package). July 30, 2009. Available from: http://www.fda.gov/downloads/Advisory Committees/CommitteesMeetingMaterials/Drugs/PsychopharmacologicDrugsAdvisoryCommittee/UCM173876.pdf. Accessed April 6, 2011.

28. US Food and Drug Administration. Drug approval package for Saphris (asenapine) tablet. Updated 18 December 2009. Available from: http:// www.accessdata.fda.gov/drugsatfda_docs/nda/2009/022117s000TOC. cfm. Accessed April 6, 2011.

29. Gerrits M, de Greef R, Peeters P. Effect of absorption site on the pharmacokinetics of sublingual asenapine in healthy male subjects. Biopharm Drug Dispos. 2010;31(5-6):351-357.

30. Shahid M, Walker GB, Zorn SH, Wong EH. Asenapine: A novel psychopharmacologic agent with a unique human receptor signature. J Psychopharmacol. 2009;23(1):65-73.

31. Shayegan DK, Stahl SM. Atypical antipsychotics: Matching receptor profile to individual patient's clinical profile. CNS Spectr. 2004; 9 Suppl 11:6-14.

32. Ballaz SJ, Akil H, Watson SJ. The 5-HT7 receptor: Role in novel object discrimination and relation to novelty-seeking behavior. Neuroscience. 2007;149(1):192-202.

33. Stahl SM. The serotonin-7 receptor as a novel therapeutic target. J Clin Psychiatry. 2010;71(11):1414-1415.

34. De Greef R, Maloney A, Olsson-Gisleskog P, Schoemaker J, Panagides J. Dopamine D2 occupancy as a biomarker for antipsychotics: Quantifying the relationship with efficacy and extrapyramidal symptoms. AAPS J. 2011;13(1):121-130.

35. Citrome L. Compelling or irrelevant? Using number needed to treat can help decide. Acta Psychiatr Scand. 2008;117(6):412-419.

36. Schering-Plough. Efficacy and safety of asenapine using an active control in subjects with schizophrenia or schizoaffective disorder. Study NCT00212784. Available from: http://www.clinicaltrials.gov/ ct2/show/record/NCT00212784. Accessed April 6, 2011.

37. Alphs L, Panagides J, Lancaster S. Asenapine in the treatment of negative symptoms of schizophrenia: Clinical trial design and rationale. Psychopharmacol Bull. 2007;40(2):41-53.

38. Schering-Plough. Efficacy and safety of asenapine compared with olanzapine in patients with persistent negative symptoms of schizophrenia. Study NCT00212836. Available from: http://www.clinicaltrials.gov/ ct2/show/record/NCT00212836. Accessed April 6, 2011.

39. Friberg LE, De Greef R, Kerbusch T, Karlsson MO. Modeling and simulation of the time course of asenapine exposure response and dropout patterns in acute schizophrenia. Clin Pharmacol Ther. 2009;86(1): 84-91.

40. Ventimiglia J, Kalali AH, Citrome L. A review of new atypical antipsychotic launches in the United States. Psychiatry (Edgmont). 2010;7(12): 13-15.

41. Citrome L. Iloperidone, asenapine, and lurasidone: A brief overview of 3 new second-generation antipsychotics. Postgrad Med. 2011; 123(2):153-162. 
42. Citrome L. Lurasidone for schizophrenia: A brief review of a new second-generation antipsychotic. Clin Schizophr Relat Psychoses. 2011; 4(4):251-257.

43. Leucht S, Corves C, Arbter D, Engel RR, Li C, Davis JM. Secondgeneration versus first-generation antipsychotic drugs for schizophrenia: A meta-analysis. Lancet. 2009;373(9657):31-41.
44. Leucht S, Komossa K, Rummel-Kluge C, et al. A meta-analysis of head-to-head comparisons of second-generation antipsychotics in the treatment of schizophrenia. Am J Psychiatry. 2009;166(2):152-163.

45. Leucht S, Arbter D, Engel RR, Kissling W, Davis JM. How effective are second-generation antipsychotic drugs? A meta-analysis of placebocontrolled trials. Mol Psychiatry. 2009;14(4):429-447.

\section{Publish your work in this journal}

Neuropsychiatric Disease and Treatment is an international, peerreviewed journal of clinical therapeutics and pharmacology focusing on concise rapid reporting of clinical or pre-clinical studies on a range of neuropsychiatric and neurological disorders. This journal is indexed on PubMed Central, the 'PsycINFO' database and CAS, and is the official

Submit your manuscript here: http://www.dovepress.com/neuropsychiatric-disease-and-treatment-journal journal of The International Neuropsychiatric Association (INA). The manuscript management system is completely online and includes a very quick and fair peer-review system, which is all easy to use. Visit http://www.dovepress.com/testimonials.php to read real quotes from published authors. 